\title{
m-almost everywhere convergence of intuitionistic fuzzy observables induced by Borel measurable function
}

\author{
Katarína Čunderlíková \\ Mathematical Institute, Slovak Academy of Sciences \\ Štefánikova 49, 81473 Bratislava, Slovakia \\ e-mail: cunderlikova.lendelova@gmail.com
}

Received: 8 February 2019

Revised: 24 April 2019

Accepted: 29 April 2019

\begin{abstract}
In paper [4] we studied the upper and the lower limits of sequence of intuitionistic fuzzy observables. We used an intuitionistic fuzzy state $\mathbf{m}$ for a definition the notion of almost everywhere convergence. We compared two concepts of $\mathbf{m}$-almost everywhere convergence. The aim of this paper is to show the connection between almost everywhere convergence in classical probability space induced by Kolmogorov construction and $\mathbf{m}$-almost everywhere convergence in intuitionistic fuzzy space. We studied the sequence of intuitionistic fuzzy observables induced by Borel measurable function.
\end{abstract}

Keywords: Intuitionistic fuzzy event, Intuitionistic fuzzy observable, Intuitionistic fuzzy state, Joint intuitionistic fuzzy observable, Product, Upper limit, Lower limit, m-almost everywhere convergence, Function of several intuitionistic fuzzy observables, Borel measurable function, Kolmogorov construction.

2010 Mathematics Subject Classification: 03B52, 60A86, 60B10.

\section{Introduction}

In [1-3] K. T. Atanassov introduced the notion of intuitionistic fuzzy sets. Then in [7] B. Riečan defined the intuitionistic fuzzy state on the family of intuitionistic fuzzy events

$$
\mathcal{F}=\left\{\left(\mu_{A}, \nu_{A}\right) ; \mu_{A}+\nu_{A} \leq 1_{\Omega}\right\},
$$


where $\mu_{A}, \nu_{A}$ are $\mathcal{S}$-measurable functions, $\mu_{A}, \nu_{A}: \Omega \rightarrow[0,1]$, as a mapping $\mathbf{m}$ from the family $\mathcal{F}$ to the set $R$ by the formula

$$
\mathbf{m}\left(\left(\mu_{A}, \nu_{A}\right)\right)=(1-\alpha) \int_{\Omega} \mu_{A} d P+\alpha\left(1-\int_{\Omega} \nu_{A} d P\right)
$$

where $P: \mathcal{S} \rightarrow[0,1]$ is a probability measure and $\alpha \in[0,1]$.

In paper [4] we defined the upper and the lower limits for sequence of intuitionistic fuzzy observables. We used an intuitionistic fuzzy state $\mathbf{m}$ for a definition the notion of almost everywhere convergence. We compared two concepts of $\mathbf{m}$-almost everywhere convergence.

In this paper we study the $\mathbf{m}$-almost everywhere convergence of sequence of intuitionistic fuzzy observables $g_{n}\left(x_{1}, \ldots, x_{n}\right): \mathcal{B}(R) \rightarrow \mathcal{F}$ given by

$$
g_{n}\left(x_{1}, \ldots, x_{n}\right)=h_{n} \circ g_{n}^{-1},
$$

where $h_{n}: \mathcal{B}\left(R^{n}\right) \rightarrow \mathcal{F}$ is the joint intuitionistic fuzzy observable of intuitionistic fuzzy observables $x_{1}, \ldots, x_{n}$ and $g_{n}: R^{n} \rightarrow R$ is a Borel measurable function. We show the connection between $\mathbf{m}$-almost everywhere convergence of this sequence of intuitionistic fuzzy observables and $P$-almost everywhere convergence of random variables in classical probability space induced by Kolmogorov construction.

Remark. Note that in a whole text we use a notation "IF" in short as the phrase "intuitionistic fuzzy."

\section{IF-events, IF-states and IF-observables}

First we start with definitions of basic notions.

Definition 2.1. Let $\Omega$ be a nonempty set. An IF-set $\mathbf{A}$ on $\Omega$ is a pair $\left(\mu_{A}, \nu_{A}\right)$ of mappings $\mu_{A}, \nu_{A}: \Omega \rightarrow[0,1]$ such that $\mu_{A}+\nu_{A} \leq 1_{\Omega}$.

Definition 2.2. Start with a measurable space $(\Omega, \mathcal{S})$. Hence $\mathcal{S}$ is a $\sigma$-algebra of subsets of $\Omega$. An IF-event is called an IF-set $\mathbf{A}=\left(\mu_{A}, \nu_{A}\right)$ such that $\mu_{A}, \nu_{A}: \Omega \rightarrow[0,1]$ are $\mathcal{S}$-measurable.

The family of all IF-events on $(\Omega, \mathcal{S})$ will be denoted by $\mathcal{F}, \mu_{A}: \Omega \longrightarrow[0,1]$ will be called the membership function, $\nu_{A}: \Omega \longrightarrow[0,1]$ be called the non-membership function.

If $\mathbf{A}=\left(\mu_{A}, \nu_{A}\right) \in \mathcal{F}, \mathbf{B}=\left(\mu_{B}, \nu_{B}\right) \in \mathcal{F}$, then we define the Łukasiewicz binary operations $\oplus, \odot$ on $\mathcal{F}$ by

$$
\begin{aligned}
& \left.\mathbf{A} \oplus \mathbf{B}=\left(\left(\mu_{A}+\mu_{B}\right) \wedge 1_{\Omega},\left(\nu_{A}+\nu_{B}-1_{\Omega}\right) \vee 0_{\Omega}\right)\right), \\
& \left.\mathbf{A} \odot \mathbf{B}=\left(\left(\mu_{A}+\mu_{B}-1_{\Omega}\right) \vee 0_{\Omega},\left(\nu_{A}+\nu_{B}\right) \wedge 1_{\Omega}\right)\right)
\end{aligned}
$$

and the partial ordering is then given by

$$
\mathbf{A} \leq \mathbf{B} \Longleftrightarrow \mu_{A} \leq \mu_{B}, \nu_{A} \geq \nu_{B}
$$


In paper we use max-min connectives defined by

$$
\begin{aligned}
& \mathbf{A} \vee \mathbf{B}=\left(\mu_{A} \vee \mu_{B}, \nu_{A} \wedge \nu_{B}\right), \\
& \mathbf{A} \wedge \mathbf{B}=\left(\mu_{A} \wedge \mu_{B}, \nu_{A} \vee \nu_{B}\right)
\end{aligned}
$$

and the de Morgan rules

$$
\begin{aligned}
& (a \vee b)^{*}=a^{*} \wedge b^{*}, \\
& (a \wedge b)^{*}=a^{*} \vee b^{*},
\end{aligned}
$$

where $a^{*}=1-a$.

Example 2.3. Fuzzy set $f: \Omega \longrightarrow[0,1]$ can be regarded as $I F$-set, if we put

$$
\mathbf{A}=\left(f, 1_{\Omega}-f\right) .
$$

If $f=\chi_{A}$, then the corresponding IF-set has the form

$$
\mathbf{A}=\left(\chi_{A}, 1_{\Omega}-\chi_{A}\right)=\left(\chi_{A}, \chi_{A^{\prime}}\right) .
$$

In this case $\mathbf{A} \oplus \mathbf{B}$ corresponds to the union of sets, $\mathbf{A} \odot \mathbf{B}$ to the intersection of sets and $\leq$ to the set inclusion.

In the IF-probability theory $[7,9]$ instead of the notion of probability, we use the notion of state.

Definition 2.4. Let $\mathcal{F}$ be the family of all IF-events in $\Omega$. A mapping $\mathbf{m}: \mathcal{F} \rightarrow[0,1]$ is called an IF-state, if the following conditions are satisfied:

(i) $\mathbf{m}\left(\left(1_{\Omega}, 0_{\Omega}\right)\right)=1, \mathbf{m}\left(\left(0_{\Omega}, 1_{\Omega}\right)\right)=0$;

(ii) if $\mathbf{A} \odot \mathbf{B}=\left(0_{\Omega}, 1_{\Omega}\right)$ and $\mathbf{A}, \mathbf{B} \in \mathcal{F}$, then $\mathbf{m}(\mathbf{A} \oplus \mathbf{B})=\mathbf{m}(\mathbf{A})+\mathbf{m}(\mathbf{B})$;

(iii) if $\mathbf{A}_{n} \nearrow \mathbf{A}\left(\right.$ i.e. $\left.\mu_{A_{n}} \nearrow \mu_{A}, \nu_{A_{n}} \searrow \nu_{A}\right)$, then $\mathbf{m}\left(\mathbf{A}_{n}\right) \nearrow \mathbf{m}(\mathbf{A})$.

Probably the most useful result in the IF-state theory is the following representation theorem ([7]):

Theorem 2.5. To each IF-state $\mathbf{m}: \mathcal{F} \rightarrow[0,1]$ there exists exactly one probability measure $P: \mathcal{S} \rightarrow[0,1]$ and exactly one $\alpha \in[0,1]$ such that

$$
\mathbf{m}(\mathbf{A})=(1-\alpha) \int_{\Omega} \mu_{A} d P+\alpha\left(1-\int_{\Omega} \nu_{A} d P\right)
$$

for each $\mathbf{A}=\left(\mu_{A}, \nu_{A}\right) \in \mathcal{F}$.

The third basic notion in the probability theory is the notion of an observable. Let $\mathcal{J}$ be the family of all intervals in $R$ of the form

$$
[a, b)=\{x \in R: a \leq x<b\} .
$$

Then the $\sigma$-algebra $\sigma(\mathcal{J})$ is denoted $\mathcal{B}(R)$ and it is called the $\sigma$-algebra of Borel sets, its elements are called Borel sets. 
Definition 2.6. By an IF-observable on $\mathcal{F}$ we understand each mapping $x: \mathcal{B}(R) \rightarrow \mathcal{F}$ satisfying the following conditions:

(i) $x(R)=\left(1_{\Omega}, 0_{\Omega}\right), x(\emptyset)=\left(0_{\Omega}, 1_{\Omega}\right)$;

(ii) if $A \cap B=\emptyset$, then $x(A) \odot x(B)=\left(0_{\Omega}, 1_{\Omega}\right)$ and $x(A \cup B)=x(A) \oplus x(B)$;

(iii) if $A_{n} \nearrow A$, then $x\left(A_{n}\right) \nearrow x(A)$.

If we denote $x(A)=\left(x^{b}(A), 1_{\Omega}-x^{\sharp}(A)\right)$ for each $A \in \mathcal{B}(R)$, then $x^{b}, x^{\sharp}: \mathcal{B}(R) \rightarrow \mathcal{T}$ are observables, where $\mathcal{T}=\{f: \Omega \rightarrow[0,1] ; f$ is $\mathcal{S}-$ measurable $\}$.

Remark 2.7. Sometimes we need to work with n-dimensional IF-observable $x: \mathcal{B}\left(R^{n}\right) \rightarrow \mathcal{F}$ defined as a mapping with the following conditions:

(i) $x\left(R^{n}\right)=\left(1_{\Omega}, 0_{\Omega}\right), x(\emptyset)=\left(0_{\Omega}, 1_{\Omega}\right)$;

(ii) if $A \cap B=\emptyset, A, B \in \mathcal{B}\left(R^{n}\right)$, then $x(A) \odot x(B)=\left(0_{\Omega}, 1_{\Omega}\right)$ and $x(A \cup B)=x(A) \oplus x(B)$;

(iii) if $A_{n} \nearrow A$, then $x\left(A_{n}\right) \nearrow x(A)$ for each $A, A_{n} \in \mathcal{B}\left(R^{n}\right)$.

If $n=1$ we simply say that $x$ is an IF-observable.

Similarly to the classical case, the following theorem can be proved ([9]).

Theorem 2.8. Let $x: \mathcal{B}(R) \longrightarrow \mathcal{F}$ be an IF-observable, $\mathbf{m}: \mathcal{F} \longrightarrow[0,1]$ be an IF-state. Define the mapping $\mathbf{m}_{x}: \mathcal{B}(R) \longrightarrow[0,1]$ by the formula

$$
\mathbf{m}_{x}(C)=\mathbf{m}(x(C))
$$

Then $\mathbf{m}_{x}: \mathcal{B}(R) \longrightarrow[0,1]$ is a probability measure.

\section{Product operation, joint IF-observable and function of several IF-observables}

In [5] we introduced the notion of product operation on the family of IF-events $\mathcal{F}$ and showed an example of this operation.

Definition 3.1. We say that a binary operation $\cdot$ on $\mathcal{F}$ is product if it satisfying the following conditions:

(i) $\left(1_{\Omega}, 0_{\Omega}\right) \cdot\left(a_{1}, a_{2}\right)=\left(a_{1}, a_{2}\right)$ for each $\left(a_{1}, a_{2}\right) \in \mathcal{F}$;

(ii) the operation - is commutative and associative; 
(iii) if $\left(a_{1}, a_{2}\right) \odot\left(b_{1}, b_{2}\right)=\left(0_{\Omega}, 1_{\Omega}\right)$ and $\left(a_{1}, a_{2}\right),\left(b_{1}, b_{2}\right) \in \mathcal{F}$, then

$$
\left(c_{1}, c_{2}\right) \cdot\left(\left(a_{1}, a_{2}\right) \oplus\left(b_{1}, b_{2}\right)\right)=\left(\left(c_{1}, c_{2}\right) \cdot\left(a_{1}, a_{2}\right)\right) \oplus\left(\left(c_{1}, c_{2}\right) \cdot\left(b_{1}, b_{2}\right)\right)
$$

and

$$
\left(\left(c_{1}, c_{2}\right) \cdot\left(a_{1}, a_{2}\right)\right) \odot\left(\left(c_{1}, c_{2}\right) \cdot\left(b_{1}, b_{2}\right)\right)=\left(0_{\Omega}, 1_{\Omega}\right)
$$

for each $\left(c_{1}, c_{2}\right) \in \mathcal{F}$;

(iv) if $\left(a_{1 n}, a_{2 n}\right) \searrow\left(0_{\Omega}, 1_{\Omega}\right),\left(b_{1 n}, b_{2 n}\right) \searrow\left(0_{\Omega}, 1_{\Omega}\right)$ and $\left(a_{1 n}, a_{2 n}\right),\left(b_{1 n}, b_{2 n}\right) \in \mathcal{F}$, then $\left(a_{1 n}, a_{2 n}\right) \cdot\left(b_{1 n}, b_{2 n}\right) \searrow\left(0_{\Omega}, 1_{\Omega}\right)$.

The following theorem provides an example of product operation for IF-events.

Theorem 3.2 ([5, Theorem 1]). The operation · defined by

$$
\left(x_{1}, y_{1}\right) \cdot\left(x_{2}, y_{2}\right)=\left(x_{1} \cdot x_{2}, y_{1}+y_{2}-y_{1} \cdot y_{2}\right)
$$

for each $\left(x_{1}, y_{1}\right),\left(x_{2}, y_{2}\right) \in \mathcal{F}$ is product operation on $\mathcal{F}$.

In [8] B. Riečan defined the notion of a joint IF-observable and proved its existence.

Definition 3.3. Let $x, y: \mathcal{B}(R) \rightarrow \mathcal{F}$ be two IF-observables. The joint IF-observable of the IF-observables $x$, y is a mapping $h: \mathcal{B}\left(R^{2}\right) \rightarrow \mathcal{F}$ satisfying the following conditions:

(i) $h\left(R^{2}\right)=\left(1_{\Omega}, 0_{\Omega}\right), h(\emptyset)=\left(0_{\Omega}, 1_{\Omega}\right) ;$

(ii) if $A, B \in \mathcal{B}\left(R^{2}\right)$ and $A \cap B=\emptyset$, then

$$
h(A \cup B)=h(A) \oplus h(B) \text { and } h(A) \odot h(B)=\left(0_{\Omega}, 1_{\Omega}\right) ;
$$

(iii) if $A, A_{1}, \ldots \in \mathcal{B}\left(R^{2}\right)$ and $A_{n} \nearrow A$, then $h\left(A_{n}\right) \nearrow h(A)$;

(iv) $h(C \times D)=x(C) \cdot y(D)$ for each $C, D \in \mathcal{B}(R)$.

Theorem 3.4 ( [8, Theorem 3.3]). For each two IF-observables $x, y: \mathcal{B}(R) \rightarrow \mathcal{F}$ there exists their joint IF-observable.

Remark 3.5. The joint IF-observable of IF-observables $x$, y from Definition 3.3 is a two-dimensional IF-observable.

If we have several IF-observables and a Borel measurable function, we can define the IFobservable, which is the function of several IF-observables. Regarding this we provide the following definition.

Definition 3.6. Let $x_{1}, \ldots, x_{n}: \mathcal{B}(R) \rightarrow \mathcal{F}$ be IF-observables, $h_{n}$ their joint IF-observable and $g_{n}: R^{n} \rightarrow R$ a Borel measurable function. Then we define the IF-observable $g_{n}\left(x_{1}, \ldots, x_{n}\right)$ : $\mathcal{B}(R) \rightarrow \mathcal{F}$ by the formula

$$
g_{n}\left(x_{1}, \ldots, x_{n}\right)(A)=h_{n}\left(g_{n}^{-1}(A)\right)
$$

for each $A \in \mathcal{B}(R)$. 


\section{Lower and upper limits, m-almost everywhere convergence}

In [4] we defined the notions of lower and upper limits for a sequence of IF-observables and showed the connection between two kinds of $\mathbf{m}$-almost everywhere convergence.

Definition 4.1. We shall say that a sequence $\left(x_{n}\right)_{n}$ of IF-observables has lim sup, if there exists an IF-observable $\bar{x}: \mathcal{B}(R) \rightarrow \mathcal{F}$ such that

$$
\bar{x}((-\infty, t))=\bigvee_{p=1}^{\infty} \bigvee_{k=1}^{\infty} \bigwedge_{n=k}^{\infty} x_{n}\left(\left(-\infty, t-\frac{1}{p}\right)\right)
$$

for every $t \in R$. We write $\bar{x}=\limsup _{n \rightarrow \infty} x_{n}$.

Note that if another IF-observable y satisfies the above condition, then $\mathbf{m} \circ y=\mathbf{m} \circ \bar{x}$.

Definition 4.2. A sequence $\left(x_{n}\right)_{n}$ of IF-observables has $\liminf _{n \rightarrow \infty}$ if there exists an IF-observable x such that

$$
\underline{x}((-\infty, t))=\bigvee_{p=1}^{\infty} \bigwedge_{k=1}^{\infty} \bigvee_{n=k}^{\infty} x_{n}\left(\left(-\infty, t-\frac{1}{p}\right)\right)
$$

for all $t \in R$. Notation: $\underline{x}=\liminf _{n \rightarrow \infty} x_{n}$.

Theorem 4.3 ( [4, Theorem 3.3]). The IF-observables $\bar{x}, \underline{x}$ from Definition 4.1 and Definition 4.2 can be expressed in the following form

$$
\begin{aligned}
& \bar{x}(A)=\left(\overline{x^{b}}(A), 1_{\Omega}-\overline{x^{\sharp}}(A)\right), \\
& \underline{x}(A)=\left(\underline{x^{b}}(A), 1_{\Omega}-\underline{x^{\sharp}}(A)\right),
\end{aligned}
$$

for each $A \in \mathcal{B}(R)$. Here $\overline{x^{b}}, \underline{x^{b}}$ are upper and lower limits of sequence $\left(x_{n}^{b}\right)_{1}^{\infty}$ of observables in tribe $\mathcal{T}$ and $\overline{x^{\sharp}}, \underline{x^{\sharp}}$ are upper and lower limits of sequence $\left(x_{n}^{\sharp}\right)_{1}^{\infty}$ of observables in tribe $\mathcal{T}$ (see [6]).

Proposition 4.1 ( [4, Proposition 3.1]). If a sequence of IF-observables $\left(x_{n}\right)_{n}$ has $\bar{x}=\limsup _{n \rightarrow \infty} x_{n}$ and $\underline{x}=\liminf _{n \rightarrow \infty} x_{n}$, then

$$
\bar{x}((-\infty, t)) \leq \underline{x}((-\infty, t)),
$$

for every $t \in R$.

Proposition 4.2 ( [4, Proposition 4.1]). A sequence $\left(x_{n}\right)_{n}$ of an IF-observables converges malmost everywhere to 0 if and only if

$$
\begin{aligned}
\mathbf{m}\left(\bigvee_{p=1}^{\infty} \bigvee_{k=1}^{\infty} \bigwedge_{n=k}^{\infty} x_{n}\left(\left(-\infty, t-\frac{1}{p}\right)\right)\right) & =\mathbf{m}\left(\bigvee_{p=1}^{\infty} \bigwedge_{k=1}^{\infty} \bigvee_{n=k}^{\infty} x_{n}\left(\left(-\infty, t-\frac{1}{p}\right)\right)\right)= \\
& =\mathbf{m}\left(0_{\mathcal{F}}((-\infty, t))\right)
\end{aligned}
$$

for every $t \in R$. 
In accordance to Proposition 4.2 we can extend the notion of $\mathbf{m}$-almost everywhere convergence in the following way.

Definition 4.4. A sequence $\left(x_{n}\right)_{n}$ of an IF-observables converges $\mathbf{m}$-almost everywhere to an IF-observable $x$, if

$$
\begin{aligned}
\mathbf{m}\left(\bigvee_{p=1}^{\infty} \bigvee_{k=1}^{\infty} \bigwedge_{n=k}^{\infty} x_{n}\left(\left(-\infty, t-\frac{1}{p}\right)\right)\right) & =\mathbf{m}\left(\bigvee_{p=1}^{\infty} \bigwedge_{k=1}^{\infty} \bigvee_{n=k}^{\infty} x_{n}\left(\left(-\infty, t-\frac{1}{p}\right)\right)\right)= \\
& =\mathbf{m}(x((-\infty, t)))
\end{aligned}
$$

for every $t \in R$.

\section{$5 \quad P$-almost everywhere convergence and m-almost everywhere convergence}

The main result of this section is given in Theorem 5.1. The main step is presented in the following proposition.

Recall, that the corresponding probability space is $\left(R^{N}, \sigma(\mathcal{C}), P\right)$, where $\mathcal{C}$ is the family of all sets of the form

$$
\left\{\left(t_{i}\right)_{i=1}^{\infty}: t_{1} \in A_{1}, \ldots, t_{n} \in A_{n}\right\},
$$

and $P$ is the probability measure determined by the equality

$$
P\left(\left\{\left(t_{i}\right)_{i=1}^{\infty}: t_{1} \in A_{1}, \ldots, t_{n} \in A_{n}\right\}\right)=\mathbf{m}\left(x_{1}\left(A_{1}\right) \cdot \ldots \cdot x_{n}\left(A_{n}\right)\right) .
$$

The corresponding projections $\xi_{n}: R^{N} \rightarrow R$ are defined by the equality

$$
\xi_{n}\left(\left(t_{i}\right)_{i=1}^{\infty}\right)=t_{n}
$$

Proposition 5.1. Let $\left(x_{n}\right)_{n}$ be a sequence of IF-observables, $\left(\xi_{n}\right)_{n}$ the sequence of corresponding projections, $g_{n}: R^{n} \rightarrow R$ be a Borel measurable functions $(n=1,2, \ldots)$. Then

$$
\begin{aligned}
P\left(\left\{u \in R^{N}\right.\right. & \left.\left.: \limsup _{n \rightarrow \infty} g_{n}\left(\xi_{1}(u), \ldots, \xi_{n}(u)\right)<t\right\}\right) \leq \\
\leq & \mathbf{m}\left(\bigvee_{p=1}^{\infty} \bigvee_{k=1}^{\infty} \bigwedge_{n=k}^{\infty} g_{n}\left(x_{1}, \ldots, x_{n}\right)\left(\left(-\infty, t-\frac{1}{p}\right)\right)\right), \\
P\left(\left\{u \in R^{N}\right.\right. & \left.\left.: \liminf _{n \rightarrow \infty} g_{n}\left(\xi_{1}(u), \ldots, \xi_{n}(u)\right)<t\right\}\right) \geq \\
\geq & \mathbf{m}\left(\bigvee_{p=1}^{\infty} \bigwedge_{k=1}^{\infty} \bigvee_{n=k}^{\infty} g_{n}\left(x_{1}, \ldots, x_{n}\right)\left(\left(-\infty, t-\frac{1}{p}\right)\right)\right) .
\end{aligned}
$$


Proof. We have

$$
\begin{aligned}
& P\left(\left\{u \in R^{N} \quad: \quad \limsup _{n \rightarrow \infty} g_{n}\left(\xi_{1}(u), \ldots, \xi_{n}(u)\right)<t\right\}\right)= \\
& =P\left(\bigcup_{p=1}^{\infty} \bigcup_{k=1}^{\infty} \bigcap_{n=k}^{\infty}\left\{u \in R^{N}: g_{n}\left(u_{1}, \ldots, u_{n}\right)<t-\frac{1}{p}\right\}\right)= \\
& =\lim _{p \rightarrow \infty} \lim _{k \rightarrow \infty} \lim _{i \rightarrow \infty} P\left(\bigcap_{n=k}^{k+i}\left(\pi_{n}^{-1}\left(g_{n}^{-1}\left(\left(-\infty, t-\frac{1}{p}\right)\right)\right)\right)\right)= \\
& =\lim _{p \rightarrow \infty} \lim _{k \rightarrow \infty} \lim _{i \rightarrow \infty} P\left(\pi_{k+i}^{-1}\left(\bigcap_{n=k}^{k+i} g_{n}^{-1}\left(\left(-\infty, t-\frac{1}{p}\right)\right)\right)\right)= \\
& =\lim _{p \rightarrow \infty} \lim _{k \rightarrow \infty} \lim _{i \rightarrow \infty} \mathbf{m}\left(h_{k+i}\left(\bigcap_{n=k}^{k+i} g_{n}^{-1}\left(\left(-\infty, t-\frac{1}{p}\right)\right)\right)\right) \leq \\
& \leq \lim _{p \rightarrow \infty} \lim _{k \rightarrow \infty} \lim _{i \rightarrow \infty} \mathbf{m}\left(\bigwedge_{n=k}^{k+i}\left(h_{k+i} \circ g_{n}^{-1}\right)\left(\left(-\infty, t-\frac{1}{p}\right)\right)\right)= \\
& =\lim _{p \rightarrow \infty} \lim _{k \rightarrow \infty} \lim _{i \rightarrow \infty} \mathbf{m}\left(\bigwedge_{n=k}^{k+i} g_{n}\left(x_{1}, \ldots, x_{n}\right)\left(\left(-\infty, t-\frac{1}{p}\right)\right)\right)= \\
& =\mathbf{m}\left(\bigvee_{p=1}^{\infty} \bigvee_{k=1}^{\infty} \bigwedge_{n=k}^{\infty} g_{n}\left(x_{1}, \ldots, x_{n}\right)\left(\left(-\infty, t-\frac{1}{p}\right)\right)\right) .
\end{aligned}
$$

The second inequality can be proved similarly.

Theorem 5.1. Let $\left(x_{n}\right)_{n}$ be a sequence of IF-observables, $\left(\xi_{n}\right)_{n}$ be the sequence of corresponding projections, $\left(g_{n}\right)_{n}$ be a sequence of Borel measurable functions $g_{n}: R^{n} \rightarrow R$. If the sequence $\left(g_{n}\left(\xi_{1}, \ldots, \xi_{n}\right)\right)_{n}$ converges $P$-almost everywhere, then the sequence $\left(g_{n}\left(x_{1}, \ldots, x_{n}\right)\right)_{n}$ converges $\mathbf{m}$-almost everywhere and

$$
\mathbf{m}\left(\limsup _{n \rightarrow \infty} g_{n}\left(x_{1}, \ldots, x_{n}\right)((-\infty, t))\right)=\mathbf{m}\left(\liminf _{n \rightarrow \infty} g_{n}\left(x_{1}, \ldots, x_{n}\right)((-\infty, t))\right)
$$

for each $t \in R$. Moreover

$$
P\left(\left\{u \in R^{N}: \limsup _{n \rightarrow \infty} g_{n}\left(\xi_{1}(u), \ldots, \xi_{n}(u)\right)<t\right\}\right)=\mathbf{m}\left(\limsup _{n \rightarrow \infty} g_{n}\left(x_{1}, \ldots, x_{n}\right)((-\infty, t))\right)
$$

for each $t \in R$.

Proof. Let the sequence $\left(g_{n}\left(\xi_{1}, \ldots, \xi_{n}\right)\right)_{n}$ converges $P$-almost everywhere, then

$$
\begin{aligned}
P\left(\left\{u \in R^{N}\right.\right. & \left.\left.: \limsup _{n \rightarrow \infty} g_{n}\left(\xi_{1}(u), \ldots, \xi_{n}(u)\right)<t\right\}\right)= \\
& =P\left(\left\{u \in R^{N}: \liminf _{n \rightarrow \infty} g_{n}\left(\xi_{1}(u), \ldots, \xi_{n}(u)\right)<t\right\}\right) .
\end{aligned}
$$

Put

$$
\begin{aligned}
& \varphi(t)=\bigvee_{p=1}^{\infty} \bigvee_{k=1}^{\infty} \bigwedge_{n=k}^{\infty} g_{n}\left(x_{1}, \ldots, x_{n}\right)\left(\left(-\infty, t-\frac{1}{p}\right)\right), \\
& \psi(t)=\bigvee_{p=1}^{\infty} \bigwedge_{k=1}^{\infty} \bigvee_{n=k}^{\infty} g_{n}\left(x_{1}, \ldots, x_{n}\right)\left(\left(-\infty, t-\frac{1}{p}\right)\right),
\end{aligned}
$$


and

$$
\eta_{n}(u)=g_{n}\left(\xi_{1}(u), \ldots, \xi_{n}(u)\right)
$$

Since $\varphi(t) \leq \psi(t)$, then

$$
\mathbf{m}(\varphi(t)) \leq \mathbf{m}(\psi(t))
$$

By Proposition 5.1 and (1) we obtain

$$
\begin{aligned}
\mathbf{m}(\psi(t)) \leq P\left(\left\{u \in R^{N}: \liminf _{n \rightarrow \infty} \eta_{n}(u)<t\right\}\right) & =P\left(\left\{u \in R^{N}: \limsup _{n \rightarrow \infty} \eta_{n}(u)<t\right\}\right) \\
& \leq \mathbf{m}(\varphi(t)) .
\end{aligned}
$$

Hence

$$
\mathbf{m}(\varphi(t))=\mathbf{m}(\psi(t))
$$

and moreover

$$
P\left(\left\{u \in R^{N}: \limsup _{n \rightarrow \infty} \eta_{n}(u)<t\right\}\right)=\mathbf{m}(\varphi(t))=\mathbf{m}(\psi(t)) .
$$

Denote the common value by

$$
F(t)=P\left(\left\{u \in R^{N}: \limsup _{n \rightarrow \infty} \eta_{n}(u)<t\right\}\right)=\mathbf{m}(\varphi(t))=\mathbf{m}(\psi(t)) .
$$

Since $\lim \sup _{n \rightarrow \infty} \eta_{n}$ is a random variable, then $F: R \rightarrow[0,1]$ is a distribution function. Evidently

$$
\begin{aligned}
\mathbf{m}\left(\bigvee_{n=1}^{\infty} \varphi(n)\right) & =\lim _{n \rightarrow \infty} \mathbf{m}(\varphi(n))=\lim _{n \rightarrow \infty} F(n)=1, \\
\mathbf{m}\left(\bigwedge_{n=1}^{\infty} \varphi(-n)\right) & =\lim _{n \rightarrow \infty} \mathbf{m}(\varphi(-n))=\lim _{n \rightarrow \infty} F(-n)=0 .
\end{aligned}
$$

Since $\mathbf{m}$ is faithful, we obtain

$$
\bigvee_{n=1}^{\infty} \varphi(n)=\left(1_{\Omega}, 0_{\Omega}\right), \bigwedge_{n=1}^{\infty} \varphi(-n)=\left(0_{\Omega}, 1_{\Omega}\right)
$$

Let $t_{n} \nearrow t$. Evidently $\varphi\left(t_{n}\right) \leq \varphi(t)$, hence

$$
\bigvee_{n=1}^{\infty} \varphi(n) \leq \varphi(t)
$$

On the other hand to each $p \in N$ there exist $j, q \in N$ such that $\left(-\infty, t-\frac{1}{p}\right) \subset\left(-\infty, t_{j}-\frac{1}{q}\right)$, hence

$$
g_{n}\left(x_{1}, \ldots, x_{n}\right)\left(\left(-\infty, t-\frac{1}{p}\right)\right) \leq g_{n}\left(x_{1}, \ldots, x_{n}\right)\left(\left(-\infty, t_{j}-\frac{1}{q}\right)\right)
$$

and therefore

$$
\bigwedge_{n=k}^{\infty} g_{n}\left(x_{1}, \ldots, x_{n}\right)\left(\left(-\infty, t-\frac{1}{p}\right)\right) \leq \bigwedge_{n=k}^{\infty} g_{n}\left(x_{1}, \ldots, x_{n}\right)\left(\left(-\infty, t_{j}-\frac{1}{q}\right)\right) \leq \varphi\left(t_{j}\right) \leq \bigvee_{j=1}^{\infty} \varphi\left(t_{j}\right) .
$$


Since the relation holds for every $k$ and $p$, we obtain

$$
\varphi(t)=\bigvee_{p=1}^{\infty} \bigvee_{k=1}^{\infty} \bigwedge_{n=k}^{\infty} g_{n}\left(x_{1}, \ldots, x_{n}\right)\left(\left(-\infty, t-\frac{1}{p}\right)\right) \leq \bigvee_{j=1}^{\infty} \varphi\left(t_{j}\right)
$$

Hence

$$
\varphi(t)=\bigvee_{j=1}^{\infty} \varphi\left(t_{j}\right)
$$

Let us summarize: $\varphi$ is non-decreasing, $\bigvee_{n=1}^{\infty} \varphi(n)=\left(1_{\Omega}, 0_{\Omega}\right), \bigwedge_{n=1}^{\infty} \varphi(-n)=\left(0_{\Omega}, 1_{\Omega}\right)$, $t_{n} \nearrow t$ implies $\varphi\left(t_{n}\right) \nearrow \varphi(t)$.

Put $y_{n}=g_{n}\left(x_{1}, \ldots, x_{n}\right)$. Using max-min connectives $\vee, \wedge$, the De Morgan rules and equality $y_{n}(A)=\left(y_{n}^{b}(A), 1_{\Omega}-y_{n}^{\sharp}(A)\right)$ we obtain

$$
\bigvee_{p=1}^{\infty} \bigvee_{k=1}^{\infty} \bigwedge_{n=k}^{\infty} y_{n}(A)=\left(\bigvee_{p=1}^{\infty} \bigvee_{k=1}^{\infty} \bigwedge_{n=k}^{\infty} y_{n}^{b}(A), 1_{\Omega}-\bigvee_{p=1}^{\infty} \bigvee_{k=1}^{\infty} \bigwedge_{n=k}^{\infty} y_{n}^{\sharp}(A)\right)
$$

for each $A \in \mathcal{B}(R)$, where $y_{n}^{b}, y_{n}^{\sharp}: \mathcal{B}(R) \rightarrow \mathcal{T}$ are observables. Put

$$
\begin{aligned}
\varphi^{b}(t) & =\bigvee_{p=1}^{\infty} \bigvee_{k=1}^{\infty} \bigwedge_{n=k}^{\infty} y_{n}^{b}\left(\left(-\infty, t-\frac{1}{p}\right)\right), \\
\varphi^{\sharp}(t) & =\bigvee_{p=1}^{\infty} \bigvee_{k=1}^{\infty} \bigwedge_{n=k}^{\infty} y_{n}^{\sharp}\left(\left(-\infty, t-\frac{1}{p}\right)\right) .
\end{aligned}
$$

Then

$$
\varphi(t)=\left(\varphi^{b}(t), 1_{\Omega}-\varphi^{\sharp}(t)\right) .
$$

Since $\varphi$ is non-decreasing, therefore $\varphi^{b}, \varphi^{\sharp}$ are non-decreasing. More by (2) we have

$$
\begin{aligned}
& \left(1_{\Omega}, 0_{\Omega}\right)=\bigvee_{n=1}^{\infty} \varphi(n)=\bigvee_{n=1}^{\infty}\left(\varphi^{b}(n), 1_{\Omega}-\varphi^{\sharp}(n)\right)=\left(\bigvee_{n=1}^{\infty} \varphi^{b}(n), 1_{\Omega}-\bigvee_{n=1}^{\infty} \varphi^{\sharp}(n)\right), \\
& \left(0_{\Omega}, 1_{\Omega}\right)=\bigwedge_{n=1}^{\infty} \varphi(-n)=\bigwedge_{n=1}^{\infty}\left(\varphi^{b}(-n), 1_{\Omega}-\varphi^{\sharp}(-n)\right)=\left(\bigwedge_{n=1}^{\infty} \varphi^{b}(-n), 1_{\Omega}-\bigwedge_{n=1}^{\infty} \varphi^{\sharp}(-n)\right) .
\end{aligned}
$$

Hence

$$
\begin{aligned}
& \bigvee_{n=1}^{\infty} \varphi^{b}(n)=1_{\Omega}, \bigwedge_{n=1}^{\infty} \varphi^{b}(-n)=0_{\Omega}, \\
& \bigvee_{n=1}^{\infty} \varphi^{\sharp}(n)=1_{\Omega}, \bigwedge_{n=1}^{\infty} \varphi^{\sharp}(-n)=0_{\Omega} .
\end{aligned}
$$

Since $t_{n} \nearrow t$ implies

$$
\left(\varphi^{b}\left(t_{n}\right), 1_{\Omega}-\varphi^{\sharp}\left(t_{n}\right)\right)=\varphi\left(t_{n}\right) \nearrow \varphi(t)=\left(\varphi^{b}(t), 1_{\Omega}-\varphi^{\sharp}(t)\right),
$$


then

$$
\begin{aligned}
\varphi^{b}\left(t_{n}\right) & \nearrow \varphi^{b}(t), \\
1_{\Omega}-\varphi^{\sharp}\left(t_{n}\right) & \searrow 1_{\Omega}-\varphi^{\sharp}(t) \Longleftrightarrow \varphi^{\sharp}\left(t_{n}\right) \nearrow \varphi^{\sharp}(t) .
\end{aligned}
$$

For fixed $\omega \in \Omega$ and arbitrary $t \in R$ put

$$
F_{\omega}^{b}(t)=\varphi^{b}(t)(\omega), F_{\omega}^{\sharp}(t)=\varphi^{\sharp}(t)(\omega) .
$$

Evidently $F_{\omega}^{b}, F_{\omega}^{\sharp}: R \rightarrow[0,1]$ are the non-decreasing functions and by (4), (6) and by (5), (7) we obtain that $F_{\omega}^{b}, F_{\omega}^{\sharp}$ are the distribution functions. Denote by $\lambda_{\omega}^{b}, \lambda_{\omega}^{\sharp}$ the corresponding Stieltjes probability measures and define $\bar{y}^{b}, \bar{y}^{\sharp}: \mathcal{B}(R) \rightarrow \mathcal{T}$ by the equalities

$$
\bar{y}^{b}(A)(\omega)=\lambda_{\omega}^{b}(A), \bar{y}^{\sharp}(A)(\omega)=\lambda_{\omega}^{\sharp}(A) .
$$

Then $\bar{y}^{b}, \bar{y}^{\sharp}$ are the observables and

$$
\begin{aligned}
& \bar{y}^{b}((-\infty, t))(\omega)=\lambda_{\omega}^{b}((-\infty, t))=F_{\omega}^{b}(t)=\varphi^{b}(t)(\omega), \\
& \bar{y}^{\sharp}((-\infty, t))(\omega)=\lambda_{\omega}^{\sharp}((-\infty, t))=F_{\omega}^{\sharp}(t)=\varphi^{\sharp}(t)(\omega),
\end{aligned}
$$

for each $\omega \in \Omega$. Using Theorem 4.3 and (8), (9) we have that there exists IF-observable $\bar{y}=$ $\limsup _{n \rightarrow} y_{n}$ given by

$$
\bar{y}((-\infty, t))=\left(\bar{y}^{b}((-\infty, t)), 1_{\Omega}-\bar{y}^{\sharp}((-\infty, t))\right)=\left(\varphi^{b}(t), 1_{\Omega}-\varphi^{\sharp}(t)\right)=\varphi(t) .
$$

The existence of IF-observable $\underline{y}=\liminf _{n \rightarrow} y_{n}=\psi$ can be proved similarly. Since $\mathbf{m}(\varphi(t))=$ $\mathbf{m}(\psi(t))$, then

$$
\mathbf{m}(\bar{y}((-\infty, t)))=\mathbf{m}(\underline{y}((-\infty, t)))
$$

for each $t \in R$ and by Definition 4.4 we have that $\left(y_{n}\right)_{n}=\left(g_{n}\left(x_{1}, \ldots, x_{n}\right)\right)_{n}$ converges $\mathbf{m}$-almost everywhere. Moreover

$$
P\left(\left\{u \in R^{N}: \limsup _{n \rightarrow \infty} \eta_{n}(u)<t\right\}\right)=\mathbf{m}(\bar{y}((-\infty, t)))
$$

for each $t \in R$.

\section{Conclusion}

The Theorem 5.1 is important for the proof of the Individual ergodic theorem in intuitionistic fuzzy case, where we work with the sequence of several IF-observables induced by the Borel function. 


\section{References}

[1] Atanassov, K. T. (1983). Intuitionistic Fuzzy Sets, VII ITKR Session, Sofia, 20-23 June 1983 (Deposed in Centr. Sci.-Techn. Library of the Bulg. Acad. of Sci., 1697/84) (in Bulgarian). Reprinted: Int. J. Bioautomation, 2016, 20(S1), S1-S6.

[2] Atannasov, K. T. (1999). Intuitionistic Fuzzy Sets: Theory and Applications, Physica Verlag, New York.

[3] Atanassov, K. T. (2012). On Intuitionistic Fuzzy Sets, Springer, Berlin.

[4] Čunderlíková, K. (2018). Upper and lower limits and $\mathbf{m}$-almost everywhere convergence of intuitionistic fuzzy observables, Notes on Intuitionistic Fuzzy Sets, 24 (4), 40-49.

[5] Lendelová, K. (2006). Conditional IF-probability, Advances in Soft Computing: Soft Methods for Integrated Uncertainty Modelling, 275-283.

[6] Riečan, B. \& Neubrunn, T. (1997). Integral, Measure and Ordering, Kluwer Academic Publishers, Dordrecht and Ister Science, Bratislava.

[7] Riečan, B. (2006). On a problem of Radko Mesiar: general form of IF-probabilities, Fuzzy Sets and Systems, 152, 1485-1490.

[8] Riečan, B. (2006). On the probability and random variables on IF events, Applied Artifical Intelligence, Proc. 7th FLINS Conf., Genova, D. Ruan et al. eds., 138-145.

[9] Riečan, B. (2012). Analysis of fuzzy logic models, Intelligent systems (V. Koleshko ed.), INTECH, 219-244. 